\title{
Clinical experience of 23 cases of adenoid cystic carcinoma of the external auditory canal
}

\author{
XIAOJUN JIANG ${ }^{1 *}$, LIFENG JIA $^{2 *}$, XUEYUAN ZHANG ${ }^{2}$, \\ CHENG ZHONG $^{2}$, FENG TANG ${ }^{2}$, XIAOLING CHEN $^{2}$ and WEI YUAN ${ }^{2}$
}

\begin{abstract}
${ }^{1}$ Department of Otolaryngology Head and Neck Surgery, Chongqing Renji Hospital, University of Chinese Academy of Sciences (Chongqing Fifth People's Hospital), Chongqing 400062; ${ }^{2}$ Department of Otolaryngology Head and Neck Surgery, The First Affiliated Hospital of The Third Military Medical University, Chongqing 400038, P.R. China
\end{abstract}

Received August 14, 2019; Accepted April 242020

DOI: $10.3892 / 01.2020 .12005$

\begin{abstract}
Adenoid cystic carcinoma (ACC) of the external auditory canal (EAC) is a rare tumor. The present study aimed to describe the clinical features and survival of patients with ACC of the EAC. The present retrospective study included 23 patients (mean age, $51.4 \pm 15.4$ years; 12 males) diagnosed with ACC of the EAC between January 2010 and September 2017 at The First Affiliated Hospital of The Third Military Medical University (Chongqing, China). The median disease course was 24 months. The presenting features were earache in 16 patients, otorrhea in 7 patients, hearing loss in 5 patients, feeling of aural fullness in 2 patients and EAC mass in 4 patients. Tumor stage was T1 in 13 patients, T2 in 3 patients, $\mathrm{T} 3$ in 3 patients and T4 in 4 patients. Among patients with T1 tumors, 5 underwent en bloc external EAC resection; 3 underwent local EAC resection; 1 underwent en bloc EAC resection and superficial parotidectomy; 1 underwent subtotal temporal bone resection for postoperative recurrence; Among patients with T4 tumors, 1 underwent extended temporal bone resection, right parotidectomy, right resection of middle cranial fossa tumors and right resection of temporomandibular joint capsule. 1 underwent subtotal temporal bone resection. Among these 15 patients who underwent surgery, 2 received postoperative radiotherapy, 1 received postoperative chemotherapy, 5 received postoperative chemo-radiotherapy, and $7 \mathrm{did}$ not receive postoperative chemo-radiotherapy. The 3- and 5-year
\end{abstract}

Correspondence to: Dr Wei Yuan, Department of Otolaryngology Head and Neck Surgery, The First Affiliated Hospital of The Third Military Medical University, 30 Gaotanyan Street, Chongqing 400038, P.R. China

E-mail:weiyuan175@sina.com

${ }^{*}$ Contributed equally

Abbreviations: ACC, adenoid cystic carcinoma; EAC, external auditory canal; $\mathrm{CK}$, cytokeratin

Key words: adenoid cystic carcinoma, external auditory canal, surgery, parotidectomy, misdiagnosis, case series cumulative survival rates of the 23 patients were $47.8 \%$ and $17.4 \%$, respectively. Survival may have been improved in patients who received postoperative chemo-radiotherapy and early diagnosis may be the key to improving survival.

\section{Introduction}

Adenoid cystic carcinoma (ACC) of the external auditory canal (EAC) is an extremely rare tumor characterized by an aggressive clinical behavior and high risk of lymphatic spread (1). Primary cancers of the EAC are rare, and most are squamous cell carcinomas (80\%), with ACC accounting for 5\% (2-5). The incidence of ACC of the EAC is low; however, it has high recurrence and distant metastasis rates (6-10).

The clinical symptoms of EAC carcinoma may be non-specific and generally manifest as hearing loss, EAC masses, otorrhea, and earache. Although early intermittent earache is the most common type of complaint in patients with EAC carcinoma, infection and otorrhea is not rare among some elderly patients $(1,11)$. Therefore, early diagnosis is often missed, and the carcinoma may be misdiagnosed as chronic otitis media, external otitis and benign neoplasms of the external ear canal (12).

Surgery is the main treatment method for ACC of the EAC, followed by radiotherapy. Nevertheless, surgery and radiotherapy are associated with substantial morbidity $(13,14)$ and a high risk of recurrence in cases of incomplete excision (4). ACC of the EAC is associated with perineural and bone invasion, and a high risk of direct intracranial extension (4). Recurrence may be observed several years after surgery, and mortality has been mainly attributed to lung metastasis $(4,15,16)$.

To the best of our knowledge, only a few reports have included $>3$ patients with ACC of the EAC (4,15-17), with other studies being reports of single cases $(11,18-26)$. Due to the rarity of the disease, the natural course and treatment of ACC of the EAC remain poorly understood, and there are no guidelines for the diagnosis and management of this malignancy. Therefore, the aim of the present study was to review the clinical features of patients with ACC of the EAC who were diagnosed and managed at a single center. It was anticipated that the results may improve the knowledge of this disease and guide diagnosis and treatment strategies to improve patient outcomes. 


\section{Materials and methods}

Study design and patients. The present retrospective study included patients diagnosed with ACC of the EAC between January 2010 and September 2017 at the Department of Otorhinolaryngology-Head and Neck Surgery, The First Affiliated Hospital of The Third Military Medical University (Chongqing, China). The present study was approved by the Ethics Committee of the Southwest Military Hospital of the Military Army Medical University. The requirement of informed consent was waived due to the retrospective nature of the study.

Clinical information of the patients was retrieved from the hospital databases. The patients were identified using 'malignant tumors of the EAC' as the search criterion. As ACC is primarily diagnosed on the basis of pathological examination (27), data retrieved using the above search criterion were then manually screened to identify patients who had a final diagnosis of ACC of the EAC. Patients diagnosed with ACC originating in the EAC (excluding ACC invading the EAC but originating from outside it) and those who were diagnosed and treated at the aforementioned hospital were included in the study. Patients with incomplete medical records were excluded from the present study. All pathologic slides were reviewed to confirm the diagnosis in all cases. Pathological examination was performed via HE staining and immunohistochemistry analysis was performed by pathologists from the Department of Pathology, The First Affiliated Hospital of the Third Military Medical University (Chongqing, China).

TNM staging. All patients were staged according to the Pittsburgh staging criteria modified by Moody et al in 2000 (28). T1N1 tumors were considered as stage 3. T2-4N1 tumors were considered as stage 4 . All stages were final stages after surgery.

Treatment. Local resection, en bloc EAC resection or subtotal temporal bone resection were performed for patients with early-stage cancer. Subtotal temporal bone resection or extended subtotal temporal bone resection were performed for patients with advanced-stage cancer. A total parotidectomy was performed for tumors that exhibited advanced invasion of the anterior wall of the EAC. A superficial parotidectomy was performed if enhancing masses were found in the parotid gland by enhanced CT. Temporomandibular joint or mandibular branch resection was also performed if imaging identified mandibular condyle or temporomandibular joint involvement. For tumors involving the meninges, the lesion was completely resected, and abdominal fat was used to fill the cavity. The facial nerves were resected if facial nerve involvement was identified during imaging or surgery. The patients received $75 \mathrm{mg} / \mathrm{m}^{2}$ docetaxel and $80 \mathrm{mg} / \mathrm{m}^{2}$ cisplatin on day 1 of each 21 -day cycle. After two cycles, local radiotherapy (33 fractions) was performed using a dose of $70 \mathrm{~Gy}$ for the GTV (tumor target area), 60 Gy for the CTV1 (high-risk clinical target area) and 54 Gy for the CTV2 (low-risk clinical target area). The patients received two cycles of chemotherapy after the end of radiotherapy.

Follow-up. Routine follow-up was performed once a month in the first year, once every 3 months in the second year, once

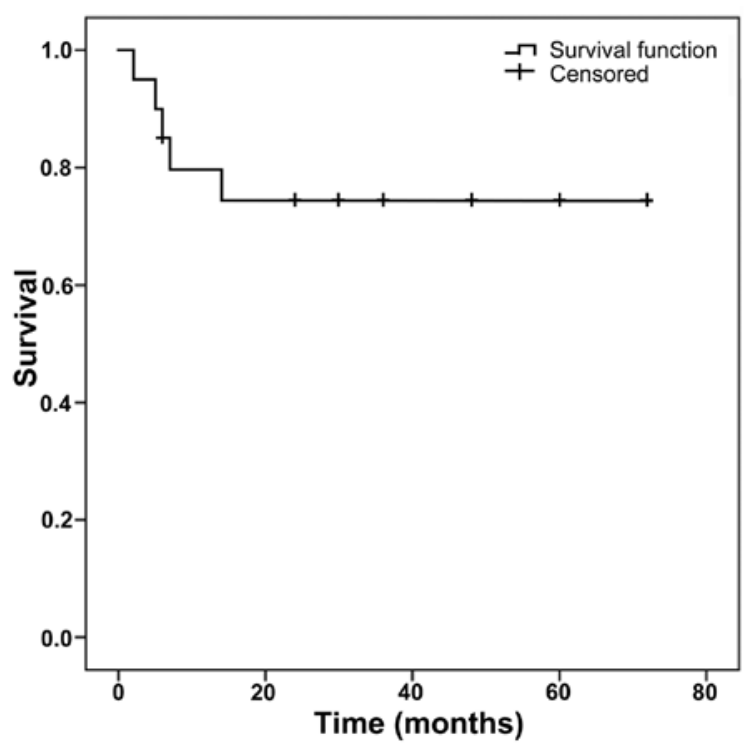

Figure 1. Overall survival of patients with adenoid cystic carcinoma of the external auditory canal.

every 6 months in the third year and once a year thereafter. The patients were instructed to visit the hospital when they felt indisposed. A biopsy was performed when a new lesion was found by imaging. Recurrence was defined as biopsy results exhibiting ACC. Follow-up was ceased on June 1, 2018.

Statistical analysis. Statistical analysis was performed using SPSS 19.0 software (IBM Corp.). The Kaplan-Meier method was used for survival analysis. Continuous variables are presented as the mean \pm standard deviation. Categorical variables are presented as numbers and percentages.

\section{Results}

Patient characteristics. Among 479 patients diagnosed with malignant tumors of the EAC during the study period, 23 patients (4.8\%) were diagnosed with ACC of the EAC and included in the present analysis. There were 12 males and 11 females. The follow-up duration ranged between 2 and 81 months (median, 39 months). A total of 3 patients were lost to follow-up (13.0\%). The age at diagnosis ranged between 31 and 78 years (mean, 51.4 \pm 15.4 years). Fig. 1 illustrates overall survival. The demographic and clinical characteristics of the patients are presented in Table I. The initial symptoms were earache in 16 patients, otorrhea in 7 patients, EAC mass in 4 patients, hearing loss in 5 patients, and a feeling of aural fullness in 2 patients (Table II). The staging of all patients was determined based on CT and MRI findings, intraoperative findings, and postoperative pathological reports. The 23 ACC of the EAC cases were classified according to the Pittsburgh staging system $(6,10)$. There were $13(56.5 \%)$ cases with $\mathrm{T} 1,3$ (13.0\%) cases with T2, 3 (13.0\%) cases with T3 and 4 (17.4\%) cases with T4 (Table III).

Fig. 2 presents the pathology images of patient 2. Fig. 2A (H\&E staining) shows the typical cribriform structure of ACC. Most of the empty spaces were round or oval pseudoglandular cavities; the lumens varied in diameter and 
Table I. Detailed patient data.

\begin{tabular}{|c|c|c|c|c|c|c|}
\hline $\begin{array}{l}\text { Patient } \\
\text { no. }\end{array}$ & $\begin{array}{c}\text { Age } \\
\text { (years)/sex }\end{array}$ & Symptom & $\begin{array}{l}\text { Pittsburgh } \\
\text { stage }\end{array}$ & $\begin{array}{l}\text { Disease course }{ }^{\mathrm{a}}, \\
\text { months }\end{array}$ & Treatment & $\begin{array}{c}\text { Follow-up and } \\
\text { outcome (months) }\end{array}$ \\
\hline 1 & $75 / \mathrm{F}$ & Earache & T3N0M0 & 1 & None & $A(36)$ \\
\hline 2 & $36 / \mathrm{F}$ & Fester, mass & T1N0M0 & 240 & En bloc EACR+CT & $\mathrm{A}(24)$ \\
\hline 3 & $40 / \mathrm{F}$ & Earache, otorrhea & T3N0M0 & 1 & En bloc EACR & $A(6)$ \\
\hline 4 & $67 / \mathrm{F}$ & Earache, otorrhea & T1N0M0 & 72 & Local EACR & LR (24); A (30) \\
\hline 5 & $34 / \mathrm{M}$ & Otorrhea & T1N0M0 & 6 & None & $\mathrm{A}(60)$ \\
\hline 6 & $46 / \mathrm{F}$ & Mass & T1N0M0 & 120 & Local EACR & LR (30); A (30) \\
\hline 7 & $75 / \mathrm{F}$ & Earache & T2N0M0 & 24 & None & DWD (5) \\
\hline 8 & $59 / \mathrm{M}$ & Earache, otorrhea & T1N0M0 & 12 & None & A (30) \\
\hline 9 & $60 / \mathrm{F}$ & Earache & T1N0M0 & 24 & None & DWD (2) \\
\hline 10 & $51 / \mathrm{M}$ & Earache & T4N1M0 & 36 & None & DWD (6) \\
\hline 11 & $50 / \mathrm{F}$ & $\begin{array}{l}\text { Hearing loss, aural } \\
\text { fullness }\end{array}$ & T4N0M0 & 6 & $\mathrm{ETBR}+\mathrm{TP}+\mathrm{RT}+\mathrm{CT}$ & $\begin{array}{l}\text { DM (12); } \\
\text { DWD (14) }\end{array}$ \\
\hline 12 & $42 / \mathrm{M}$ & Earache & T4N0M0 & 24 & None & DWD (7) \\
\hline 13 & $62 / \mathrm{M}$ & Mass, hearing loss & T1N0M0 & 11 & Local EACR+RT & LFU (3) \\
\hline 14 & $61 / \mathrm{M}$ & $\begin{array}{l}\text { Earache, hearing loss, } \\
\text { otorrhea }\end{array}$ & T3N0M0 & 36 & None & LFU (3) \\
\hline 15 & $32 / \mathrm{F}$ & Earache, hearing loss & T1N0M0 & 84 & En bloc $\mathrm{EACR}+\mathrm{RT}+\mathrm{CT}$ & A (48) \\
\hline 16 & $31 / \mathrm{M}$ & Aural fullness & T1N0M0 & 2 & En bloc EACR+RT & A (36) \\
\hline 17 & $78 / \mathrm{M}$ & Otorrhea, earache, mass & T2N0M0 & 120 & $\mathrm{RM}$ & $\mathrm{A}(48)$ \\
\hline 18 & $42 / \mathrm{F}$ & Earache & T1N0M0 & 8 & $\begin{array}{l}\text { En bloc EACR+SP+ } \\
\text { RT+CT }\end{array}$ & A (18) \\
\hline 19 & $37 / \mathrm{F}$ & Earache & T1N0M0 & 36 & En bloc $\mathrm{EACR}+\mathrm{RT}+\mathrm{CT}$ & $\mathrm{A}(72)$ \\
\hline 20 & $47 / \mathrm{M}$ & $\begin{array}{l}\text { Earache, facioplegia, } \\
\text { vertigo }\end{array}$ & T4N0M0 & 84 & $\mathrm{STBR}+\mathrm{RT}+\mathrm{CT}$ & $\mathrm{A}(30)$ \\
\hline 21 & $65 / \mathrm{M}$ & Hearing loss, otorrhea & T2N0M0 & 24 & $\mathrm{RM}$ & LFU (3) \\
\hline 22 & $40 / \mathrm{M}$ & Earache & T1N0M0 & 12 & En bloc EACR & $A(60)$ \\
\hline 23 & $52 / \mathrm{M}$ & Earache & T1N0M0 & 36 & STBR & A (24) \\
\hline
\end{tabular}

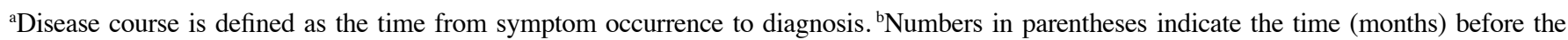
outcome occurred. F, female; M, male; RM, radical mastoidectomy; EACR, external auditory canal resection; SP, superficial parotidectomy; STBR, subtotal temporal bone resection; ETBR, extended temporal bone resection; TP, total parotidectomy; RT, radiotherapy; CT, chemotherapy; A, alive; LR, local/regional recurrence; DM, distant metastases; DWD, dead with disease; DUC, dead with unknown cause; LFU, lost to follow-up.

Table II. Clinical symptoms.

\begin{tabular}{lrc}
\hline Symptoms & Cases & Percentage \\
\hline External auditory canal masses & 4 & 11.1 \\
Earache & 16 & 44.4 \\
Otorrhea & 7 & 19.4 \\
Facioplegia & 1 & 2.7 \\
Hearing loss & 5 & 13.9 \\
Ear aural fullness & 2 & 5.6 \\
Vertigo & 1 & 2.7 \\
\hline
\end{tabular}

were uniformly basophilic. Fig. $2 \mathrm{~B}$ and $\mathrm{C}$ illustrate the immunohistochemistry results for CD117 and CK, respectively. CD117 was localized on the glandular epithelial cell
Table III. Tumor staging.

\begin{tabular}{lrc}
\hline TNM staging & Cases & Percentage \\
\hline Tumor size & & \\
T1 & 13 & 56.5 \\
T2 & 3 & 13.0 \\
T3 & 3 & 13.0 \\
T4 & 4 & 17.4 \\
Lymph node metastasis & & \\
N0 & 22 & 95.7 \\
N1 & 1 & 4.3 \\
Distant metastasis & & \\
M0 & 23 & 0 \\
M1 & 0 & \\
\hline
\end{tabular}

TNM, tumor-node-metastasis. 

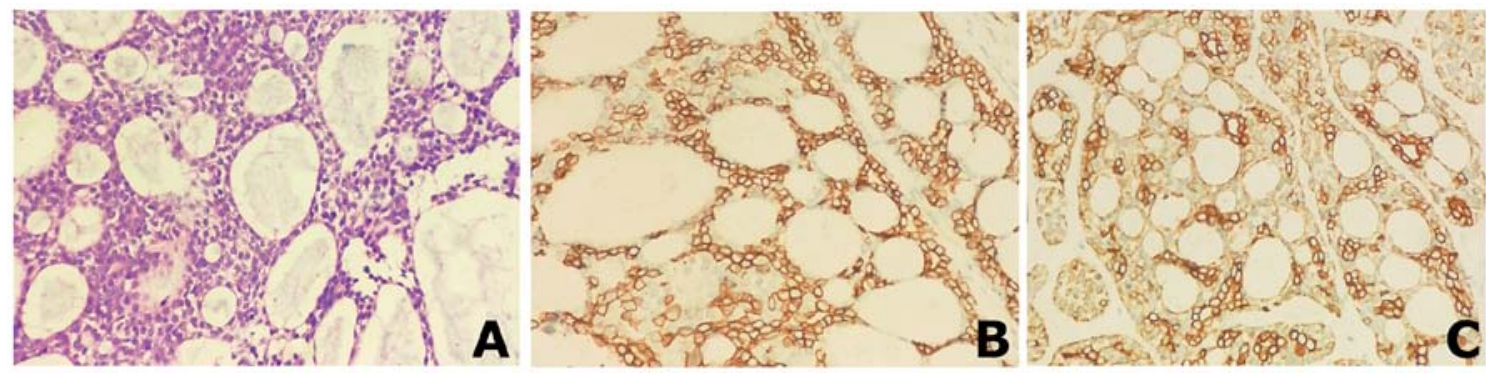

Figure 2. Pathology images of patient no. 2. (A) Hematoxylin and eosin staining revealing the typical cribriform structure of adenoid cystic carcinoma. Most of the empty spaces were round or oval pseudo-glandular cavities; the gland lumens varied in diameter and were uniformly basophilic. (B) Immunohistochemistry for CD177. CD117 was localized on the cell membrane of glandular epithelial cells. (C) Immunohistochemistry for cytokeratin. Cytokeratin was localized in the cytoplasm. Magnification, $\mathrm{x} 400$.
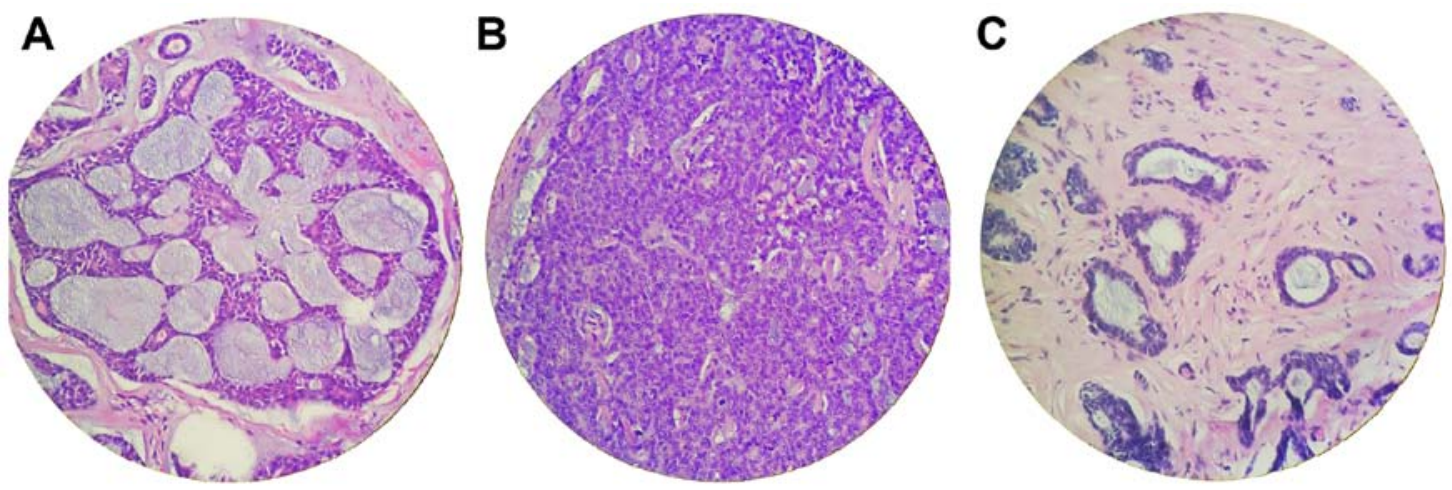

Figure 3. Pathology images showing three types of ACC. (A) Section stained with hematoxylin and eosin showing the typical cribriform structure of ACC. Most of the empty spaces were round or oval pseudo-glandular cavities; the gland lumens varied in diameter and were uniformly basophilic. (B) Section stained with hematoxylin and eosin showing the typical solid structure of ACC. The tumor cells formed large solid epithelial cell islands and clumps. Tumor cells were mainly myoepithelial cells, which were rich and dense, with deeply stained nuclei and rough nuclear chromatin. The heteromorphism of the nuclei was obvious. (C) Section stained with hematoxylin and eosin showing the typical tubular structure of ACC. The neoplastic cells formed multiple tubular, strip-like adenoid structures. The glandular duct was composed of small cuboidal epithelial cells and myoepithelial cells. Hyaline basement membranoid deposits could be observed around the ducts. Magnification, x400. ACC, adenoid cystic carcinoma.

membrane, whereas CK was localized in the cytoplasm. Both markers were strongly positive. Fig. 3 illustrates representative images of the three subtypes of ACC. The illustrated pathological images were obtained from the Department of Pathology, The First Affiliated Hospital of the Third Military Medical University (Chongqing, China).

Among the 23 patients, 8 patients declined surgery, and the remaining 15 patients underwent surgery under general anesthesia. Among these 15 patients, 1 patient had T4 disease and underwent subtotal temporal bone resection, another patient with T4 underwent extended temporal bone resection, right parotidectomy, right resection of middle cranial fossa tumors and right resection of temporomandibular joint capsule. One patient with T3 underwent en bloc EAC resection and 2 patients with $\mathrm{T} 2$ underwent radical mastoidectomy. The remaining 10 patients had $\mathrm{T} 1$ disease, of which 5 patients underwent en bloc EAC resection, 3 underwent local EAC resection, 1 underwent en bloc resection of EAC and superficial parotidectomy, and 1 underwent subtotal temporal bone resection for postoperative recurrence after treatment in another hospital. Among these 15 surgical patients, 2 received postoperative radiotherapy alone, 1 received postoperative chemotherapy alone, 5 received postoperative chemo-radiotherapy and 7 did not receive postoperative chemo-radiotherapy (Tables IV and V).
Of the 23 patients, 5 patients died due to the primary disease, including 1 case with T1, 1 case with T2, and 3 cases with T4 disease. Among the living patients, 13 patients were healthy and exhibited no signs of recurrence, 2 patients exhibited recurrence but were alive. Furthermore, 3 patients were lost to follow-up. The 3- and 5-year cumulative survival rate were calculated via Kaplan-Meier survival curve analysis and were $47.8 \%$ and $17.4 \%$, respectively (Table I and Fig. 1).

The present study demonstrated that the 5-year cumulative survival rate was $40 \%$ in patients treated using a combination of surgery and radio-chemotherapy, $14.3 \%$ in patients treated with surgery alone, $0 \%$ in patients treated with a combination of surgery and radiotherapy and $0 \%$ in patients treated with a combination of surgery and chemotherapy (Fig. 1).

Recurrence and survival. Among the 23 patients, $2(8.7 \%)$ had disease recurrence, and both underwent local EACR without postoperative chemo-radiotherapy. No further treatment was carried out after recurrence, but the patients were still alive at the time of writing. In the present study, $5 / 23$ patients $(21.7 \%)$ died: 1 patient with $\mathrm{T} 1$ disease, 1 with $\mathrm{T} 2$ disease and 3 with T4 disease. The majority (4/5) of the patients who died had declined treatment and died of their primary tumor after discharge. One patient who underwent extended temporal bone resection, right parotidectomy, right resection of middle cranial 
Table IV. Treatment in relation to clinical stage.

\begin{tabular}{lccccc}
\hline Stage & $\begin{array}{c}\text { Surgery } \\
\text { alone }\end{array}$ & Surgery + radiotherapy & Surgery + chemotherapy & Surgery + chemo-radiotherapy & $\begin{array}{c}\text { Conservative } \\
\text { treatment }\end{array}$ \\
\hline T1 & 4 & 2 & 1 & 3 & 3 \\
T2 & 2 & 0 & 0 & 0 & 1 \\
T3 & 1 & 0 & 0 & 0 & 2 \\
T4 & 0 & 0 & 0 & 2 & 2 \\
Total & 7 & 2 & 1 & 5 & 8 \\
\hline
\end{tabular}

Table V. Surgical methods in relation to clinical stage.

\begin{tabular}{lcccccc}
\hline Stage & RM & $\begin{array}{c}\text { Local } \\
\text { EACR }\end{array}$ & $\begin{array}{c}\text { En bloc } \\
\text { EACR }\end{array}$ & $\begin{array}{c}\text { En bloc } \\
\text { EACR+SP }\end{array}$ & $\begin{array}{c}\text { STBR } \\
\text { ETBR+ } \\
\text { TP }\end{array}$ \\
\hline T2 & 0 & 3 & 5 & 1 & 1 & 0 \\
T3 & 2 & 0 & 0 & 0 & 0 & 0 \\
T4 & 0 & 0 & 1 & 0 & 0 & 0 \\
Total & 2 & 3 & 7 & 0 & 1 & 1 \\
\hline
\end{tabular}

RM, radical mastoidectomy; EACR, external auditory canal resection; SP, superficial parotidectomy; STBR, subtotal temporal bone resection; ETBR, extended temporal bone resection; TP, total parotidectomy.

fossa tumors and right resection of the temporomandibular joint capsule received postoperative chemo-radiotherapy and died of systemic metastasis 14 months after surgery (Fig. 1).

Associations among disease course, lesion stage and curative effect. As presented in Table I the disease course (time from symptom occurrence to diagnosis) was 1-240 months (median, 24 months). Among the patients with a disease course $\leq 24$ months, there were 7 patients with $\mathrm{T} 1$ disease $(5$ survivors and 1 lost to follow-up and 1 dead), 2 patients with T2 disease (1 lost to follow-up and 1 dead), 2 patient with T3 disease (alive) and 2 patient with T4 disease (dead). Among patients with a disease course $>24$ months, there were 6 patients with T1 disease (alive), 1 patient with T2 disease (alive), 1 patient with T3 disease (lost to follow-up) and 2 patients with T4 disease ( 1 dead and 1 alive). For patients with a disease course $>24$ months, the initial symptoms were earache, hearing loss and otorrhea, but these symptoms did not attract attention or were misdiagnosed at other hospitals as benign masses in the EAC and chronic otitis media, which delayed the diagnosis and treatment.

Misdiagnosed cases. A total of 2 patients were misdiagnosed with otitis media before surgery (initial symptoms of otorrhea and hearing loss). Additionally, 2 patients were misdiagnosed preoperatively with benign masses of the EAC; 1 patient had symptoms of earache and itchy ears and 1 patient had EAC masses for $>10$ years. No biopsy was performed prior to surgery, and radical mastoidectomy and en bloc resection of the EAC masses were performed in the 2 patients. Postoperative pathology confirmed a diagnosis of ACC. Further treatment was recommended, but none of the 4 patients received surgical treatment or radiotherapy. At the end of the follow-up period, 2 patients were still alive after en bloc mass resection and the 2 patients misdiagnosed with otitis media were lost to follow-up.

\section{Discussion}

The present case series describes the clinical features and management of ACC of the EAC. Although several studies have reported patients with $\mathrm{ACC}$ of the EAC, most of these have been reports of single cases $(11,18-26)$, with only a few including more than three patients $(4,15-17)$. The present study included 23 patients from a single center and, to the best of our knowledge, is the largest reported series to date.

ACC is a rare form of adenocarcinoma that arises from glandular tissue. Although the origin of primary ACC of the EAC remains controversial, it has been suggested that the malignancy arises from eccrine sweat glands, ectopic salivary glands, or ceruminous glands (11). The differential diagnosis of ACC of the EAC includes adenoma; papilloma; tuberculosis; and tumors such as squamous cell carcinoma, adenocarcinoma, basal cell carcinoma, and mucoepidermoid carcinoma (11). Immunohistochemistry studies of salivary gland ACC demonstrated that carcinoembryonic antigen (CEA) is expressed in some cells (29), and p53 expression is associated with poor outcomes (30). However, immunohistochemistry data for p53 and CEA are limited for ACC of the EAC.

The association between ACC and age or sex is controversial. Triantafillidou et al (29) reported a female predilection for ACC, whereas De Lucia et al (30) reported a male predilection. The present study found that the number of males with ACC of the EAC was slightly higher than the number of females, and the average age of onset was approximately 50 years.

Fliss et al (31) suggested that earache is the most common clinical symptom of ACC of the EAC. This is concurrent with the findings of the present study, in which the most common initial symptom was earache (reported by $43.2 \%$ of patients), followed by otorrhea, hearing loss, and ear mass. Since these symptoms are non-specific, more attention must be paid to them when they are chronic, recurrent or aggravated, or if an ear mass exhibits progressive growth.

Early diagnosis of the disease is crucial to improve the survival of patients with ACC of the EAC; however, the misdiagnosis rate is relatively high. In the present study, 4 out of 23 patients (17.4\%) were misdiagnosed. This high misdiag- 
nosis rate could be explained by the low disease incidence rate and a resultant lack of awareness of the disease. Additionally, because there are no typical symptoms, the atypical symptoms of ACC of the EAC, such as earache, otorrhea, and hearing loss, are often misdiagnosed as external otitis and otitis media. Furthermore, although biopsy must be performed for suspected cases, awareness of the necessity of a biopsy is low among physicians.

The surgical principle for the tumor is the same as that for most malignant tumors, namely, complete curative resection. The surgical methods include local EAC resection, en bloc EAC resection, subtotal temporal bone resection, and temporal bone resection. In the present study, two patients with local resection of the EAC exhibited recurrence, indicating that even early-stage disease can invade the surrounding tissues, and that local mass resection does not guarantee a safe margin and can result in recurrence. Garden et al (32) suggested that a positive margin is a vital factor leading to poor prognosis. Due to the lack of treatment guidelines, the recommendations of representative literature, including case series, were mainly followed for the treatment of patients in the present study. Some authors recommended early extended resection $(4,10)$. In addition to removing local tumors, the surrounding tissues such as the cartilaginous and osseous parts of the EAC, middle ear, and even the whole temporal bone and parotid glands should be removed. For ACC of the EAC, a large-area total resection of the EAC is recommended to remove related cartilage, mastoid process, and middle ear. If the tumor is observed to invade the middle ear and temporal bone, or involve the facial nerve during preoperative examinations or operative procedure, subtotal resection or total resection of the temporal bone can be performed, and the corresponding dura mater or the temporomandibular joint can be removed if necessary. Some authors recommend the lateral resection of the temporal bone for patients with $\mathrm{T} 1$ and $\mathrm{T} 2$ stages, and subtotal resection of the temporal bone and infratemporal fossa for those with T3 and $\mathrm{T} 4$ stages (28). The range for lateral resection of the temporal bone includes cartilaginous and osseous parts of the EAC, tympanic bone, tympanic membrane, malleus, and incus. The medial side is limited to the level of the incudostapedial joint and lateral facial nerve (33). Subtotal resection of the temporal bone is based on the lateral resection of the temporal bone to expand the resection inward and remove the inner ear, but retaining the apex of the petrous temporal bone (3). Although there are differences in the treatment methods among studies, the consensus is that the appropriate surgical methods should be based on the invaded sites and stages of tumors of the EAC to achieve the goal of complete tumor resection (28).

Additionally, the treatment of the parotid gland is controversial. Some authors consider that superficial parotidectomy is suitable for all patients with ACC of the EAC, even in the early stages (12). The EAC and parotid gland have a close histological association. Additionally, the Santorini cracks provide a way for invading the parotid gland (12). In the present series, there was no difference in survival between patients with T1 and T4 disease. Nevertheless, it is recommended that patients with early disease undergo superficial parotidectomy at the same time as lesion resection because the lack of recurrence observed in the present study may be caused by a combination of factors such as a short follow-up and a small sample size. The parotid gland can be partially removed to ensure safety. If the parotid gland is markedly invaded, it can be completely removed (34).

Moffat et al (35) suggested that radiotherapy can destroy subclinical tumor foci and increase surgical efficacy. Radiotherapy may be particularly effective for advanced tumors when it is difficult to achieve safe margins. Additionally, Chen et al (36) suggested that postoperative radiotherapy with $>60$ Gy radiation can effectively prevent postoperative recurrence of ACC of the head and neck. Silverman et al (37) suggested that postoperative radiotherapy is required for patients with advanced clinical disease, whereas patients with negative margins after the first surgery and low clinical stage do not require radiotherapy. It has been suggested that chemotherapy does not affect the survival of patients with ACC (38). The results of the present study indicate that the survival rate of the combined treatment of surgery and chemoradiotherapy is higher than surgery alone or postoperative isolated radiotherapy or chemotherapy. Therefore, the survival rate was notably higher for patients who received surgery and radiochemotherapy than for patients who were not given all three treatment modalities.

In the present study, two patients exhibited recurrence during follow-up and one patient with T4 disease died of intracranial and pulmonary metastases 14 months after surgery. Distant metastasis is more likely to occur in the lungs, followed by bone, kidney, and brain (34). Therefore, close attention should be paid to the lungs, brain, kidneys, and other sites prone to metastasis during follow-up.

The present study has certain limitations. First, the sample size was small due to the low incidence of ACC of the EAC and because this was a single-center study. In addition, as all cases were from a single center, this may lead to some bias based on the diagnostic experience of the radiologists and pathologists, as well as the experience of the surgeons and oncologists. Second, there are three generally recognized histological patterns of ACC (11); however, these patterns were not consistently described in the pathology reports. Third, the p53 and CEA immunohistochemistry data were not available for the patients in the present study. Thus, it was not possible to evaluate these factors. Fourth, positron emission tomography (PET)-CT scans were not performed during follow-up to detect the occurrence of distant metastasis. This was partly due to PET-CT scans being unaffordable for a number of the patients as they were not covered by medical insurance. Therefore, it was not possible for the current retrospective analysis to systematically evaluate the occurrence of distant metastasis. Fifth, only routine follow-up data were available for the current retrospective analysis, and $17 \%$ of the patients were lost to follow-up. Patients may move residence, die at another hospital, or simply refuse follow-up. A prospective study would be difficult to carry out because of the rarity of the disease, but a multicenter retrospective study could be performed to refine the results of the present study.

Patients with a complaint of an EAC mass, EAC pain, and otorrhea should be examined comprehensively with CT or MRI and biopsy, if necessary. The initial surgical treatment should be tumor resection with wide margins and superficial parotidectomy. Frozen-section examination should be performed to ensure a negative margin. The survival rate of patients after postoperative chemoradiotherapy was higher than that of patients without chemoradiotherapy. Early diagnosis may be 
the key to improving survival. Nevertheless, it is noteworthy to highlight that a 75-year-old female patient with T3 disease refused surgery and chemoradiotherapy and survived with the tumor for at least 51 months, since the patient was still alive when the present report was written. Although this phenomenon has been observed before, the specific reasons remain to be determined (39).

Therefore, early identification of symptoms, performance of necessary imaging, and timely biopsy are key to reducing misdiagnosis and improving the survival rate. It is recommended to expand the tumor for the first operation and perform parotidectomy for the superficial parotid gland. Pathological examination must be performed during the surgery to ensure a negative margin. For patients with doubtful negative margins, postoperative radiotherapy and chemotherapy can be considered to reduce recurrence and improve survival. The lack of unified staging and treatment guidelines coupled with the low incidence of the disease and few studies requires further investigations on the specificity, biological behavior and optimal treatment plan of the tumor.

\section{Acknowledgements}

Not applicable.

\section{Funding}

No funding was received.

\section{Availability of data and materials}

The datasets used and/or analyzed during the present study are available from the corresponding author upon reasonable request.

\section{Authors' contributions}

XJ participated in case collection, follow-up, analysis and drafted the initial manuscript and was responsible for the study concept and design. LJ participated in data analysis and interpretation of the data. $\mathrm{XZ}$ and $\mathrm{CZ}$ made significant contributions to the data acquisition, data analyses and interpretation. FT and XC participated in patient follow-up. WY performed the surgeries and participated in formulating the treatment plan. WY also helped prepare the final version of the manuscript to be submitted. All the authors made significant contributions to the study design, data analysis, interpretation and drafting of the paper. All the authors have read and approved of the final manuscript for publication.

\section{Ethics approval and consent to participate}

The present study was approved by the Ethics Committee of The First Affiliated Hospital of The Third Military Medical University (Chongqing, China; approval no. KY2020113). The requirement for informed consent was waived due to the retrospective nature of the study.

\section{Patient consent for publication}

Not applicable.

\section{Competing interests}

The authors declare that they have no competing interests.

\section{References}

1. Gu FM, Chi FL, Dai CF, Chen B and Li HW: Surgical outcomes of 43 cases with adenoid cystic carcinoma of the external auditory canal. Am J Otolaryngol 34: 394-398, 2013.

2. Conley $J$ and Schuller DE: Malignancies of the ear. Laryngoscope 86: 1147-1163, 1976.

3. Moore MG, Deschler DG, McKenna MJ, Varvares MA and Lin DT: Management outcomes following lateral temporal bone resection for ear and temporal bone malignancies. Otolaryngol Head Neck Surg 137: 893-898, 2007.

4. Dong F, Gidley PW, Ho T, Luna MA, Ginsberg LE and Sturgis EM: Adenoid cystic carcinoma of the external auditory canal. Laryngoscope 118: 1591-1596, 2008.

5. Chang CH, Shu MT, Lee JC, Leu YS, Chen YC and Lee KS: Treatments and outcomes of malignant tumors of external auditory canal. Am J Otolaryngol 30: 44-48, 2009.

6. Arriaga M, Curtin H, Takahashi H, Hirsch BE and Kamerer DB: Staging proposal for external auditory meatus carcinoma based on preoperative clinical examination and computed tomography findings. Ann Otol Rhinol Laryngol 99: 714-721, 1990.

7. Shih L and Crabtree JA: Carcinoma of the external auditory canal: An update. Laryngoscope 100: 1215-1218, 1990.

8. Kuhel WI, Hume CR and Selesnick SH: Cancer of the external auditory canal and temporal bone. Otolaryngol Clin North Am 29: 827-852, 1996.

9. Testa JR, Fukuda Y and Kowalski LP: Prognostic factors in carcinoma of the external auditory canal. Arch Otolaryngol Head Neck Surg 123: 720-724, 1997.

10. Nyrop $\mathrm{M}$ and Grontved A: Cancer of the external auditory canal. Arch Otolaryngol Head Neck Surg 128: 834-837, 2002.

11. Liu SC, Kang BH, Nieh S, Chang JL and Wang CH: Adenoid cystic carcinoma of the external auditory canal. J Chin Med Assoc 75: 296-300, 2012.

12. Zhang T, Dai C and Wang Z: The misdiagnosis of external auditory canal carcinoma. Eur Arch Otorhinolaryngol 270: $1607-1613,2013$.

13. Kwok HC, Morton RP, Chaplin JM, McIvor NP and Sillars HA: Quality of life after parotid and temporal bone surgery for cancer. Laryngoscope 112: 820-833, 2002.

14. Wang CC: Radiation therapy in the management of carcinoma of the external auditory canal, middle ear, or mastoid. Radiology 116: 713-715, 1975.

15. Pulec JL, Parkhill EM and Devine KD: Adenoid cystic carcinoma (Cylindroma) of the external auditory canal. Trans Am Acad Ophthalmol Otolaryngol 67: 673-694, 1963.

16. Perzin KH, Gullane P and Conley J: Adenoid cystic carcinoma involving the external auditory canal. A clinicopathologic study of 16 cases. Cancer 50: 2873-2883, 1982.

17. Choi JY, Choi EC, Lee HK, Yoo JB, Kim SG and Lee WS: Mode of parotid involvement in external auditory canal carcinoma. J Laryngol Otol 117: 951-954, 2003.

18. O'Neill PB and Parker RA: Sweat gland tumours (ceruminomata) of external auditory meatus. J Laryngol Otol 71: 824-831, 1957.

19. Cankar V and Crowley H: Tumors of Ceruminous Glands: A Clinicopathological Study of 7 Cases. Cancer 17: 67-75, 1964.

20. Batsakis JG, Hardy GC and Hishiyama RH: Ceruminous gland tumors. Arch Otolaryngol 86: 66-69, 1967.

21. Neldner KH: Ceruminoma. Arch Dermatol 98: 344-348, 1968.

22. Ahmed GM and Nath DK: Ceruminous gland tumour (a case report). J Laryngol Otol 102: 346-349, 1988.

23. Goldman NC: Adenoid cystic carcinoma of the external auditory canal. Otolaryngol Head Neck Surg 106: 214-215, 1992.

24. Mohan H, Handa U, Amanjit, Kotwal SA and Dass A: Adenoid cystic carcinoma of the external auditory canal. A case report with diagnosis by fine needle aspiration. Acta Cytol 47: 792-794, 2003.

25. Buda-Nowak A, Swiader M, Puskulluoglu M, Dyduch G, Krupinski M and Krzemieniecki K: Adenoid cystic carcinoma of the external auditory canal with metastases to lymph nodes and lungs-problematic diagnosis and treatment based on a case report. Przegl Lek 72: 383-386, 2015.

26. Nayak SP, Walke VA, Helwatkar SB and Bobhate SK: Adenoid cystic carcinoma of the external auditory canal: Report of two cases. Indian J Pathol Microbiol 52: 540-542, 2009. 
27. Shi $X$ and Dai C: Current status of clinical diagnosis and treatment of temporal bone malignant tumors. Chinese J Otol 17: 311-316, 2019 (In Chinese).

28. Moody SA, Hirsch BE and Myers EN: Squamous cell carcinoma of the external auditory canal: An evaluation of a staging system. Am J Otol 21: 582-588, 2000.

29. Triantafillidou K, Dimitrakopoulos J, Iordanidis F and Koufogiannis D: Management of adenoid cystic carcinoma of minor salivary glands. J Oral Maxillofac Surg 64: 1114-1120, 2006.

30. De Lucia A, Gambardella T, Carra P and Motta G: A case of highly aggressive adenoid cystic carcinoma of the external auditory canal. Acta Otorhinolaryngol Ital 24: 354-356, 2004.

31. Fliss DM, Kraus M and Tovi F: Adenoid cystic carcinoma of the external auditory canal. Ear Nose Throat 69: 635, 638-639, 642 passim, 1990.

32. Garden AS, Weber RS, Morrison WH, Ang KK and Peters LJ: The influence of positive margins and nerve invasion in adenoid cystic carcinoma of the head and neck treated with surgery and radiation. Int J Radiat Oncol Biol Phys 32: 619-626, 1995.

33. Yu YF, Zhang R and Dai CF: The study on the en bloc resection of the external auditory canal to treat external auditory canal carcinoma in the early stage. Lin Chung Er Bi Yan Hou Tou Jing Wai Ke Za Zhi 7: 313-315, 2009 (In Chinese).

34. Liu MB, Zhou QY, Wu WM, Wang JL, Liu LF and Huang DL: A review of 24 cases of external auditory canal adenoid cystic carcinoma. Chin J Otol 7, 2009 (In Chinese).
35. Moffat DA, Wagstaff SA and Hardy DG: The outcome of radical surgery and postoperative radiotherapy for squamous carcinoma of the temporal bone. Laryngoscope 115: 341-347, 2005.

36. Chen AM, Bucci MK, Weinberg V, Garcia J, Quivey JM, Schechter NR, Phillips TL, Fu KK and Eisele DW: Adenoid cystic carcinoma of the head and neck treated by surgery with or without postoperative radiation therapy: Prognostic features of recurrence. Int J Radiat Oncol Biol Phys 66: 152-159, 2006.

37. Silverman DA, Carlson TP, Khuntia D, Bergstrom RT, Saxton J and Esclamado RM: Role for postoperative radiation therapy in adenoid cystic carcinoma of the head and neck. Laryngoscope 114: 1194-1199, 2004.

38. Cristalli G, Manciocco V, Pichi B, Marucci L, Arcangeli G, Telera S and Spriano G: Treatment and outcome of advanced external auditory canal and middle ear squamous cell carcinoma. J Craniofac Surg 20: 816-821, 2009.

39. Zhao FF, Wang JL, Wu WM, Huang DL, Dai P, Yang SM, Han WJ and Han DY: Clinical analysis of adenoid cystic carcinoma of external auditory canal. Zhonghua Er Bi Yan Hou Tou Jing Wai Ke Za Zhi 44: 444-448, 2009 (In Chinese).

(i) $\odot$ This work is licensed under a Creative Commons Attribution-NonCommercial-NoDerivatives 4.0 International (CC BY-NC-ND 4.0) License. 